\title{
Progress toward BLISS, the background-limited infrared-submillimeter spectrograph for SPICA
}

\author{
C.M. Bradford ${ }^{a, b}$, A. Beyer ${ }^{a, b}$, M. Kenyon ${ }^{a}$, M.C. Runyan ${ }^{b}$, B. Bumble ${ }^{a}$, P. Echternach ${ }^{a}$, \\ T. Prouvé ${ }^{b}$, W.A. Holmes ${ }^{a}$, J.J Bock ${ }^{a, b}$, K. Irwin ${ }^{c}$ \\ ${ }^{a}$ Jet Propulsion Laboratory, California Institute of Technology, Pasadena, CA; \\ ${ }^{b}$ California Institute of Technology, Pasadena, CA; \\ ${ }^{c}$ National Institute of Standards and Technology, Boulder, CO'
}

\begin{abstract}
We are developing the Background-Limited Infrared-Submillimeter Spectrograph (BLISS) for SPICA to provide a breakthrough capability for far-IR survey spectroscopy. The 3.2-meter, actively-cooled $(\mathrm{T}<6 \mathrm{~K}) \mathrm{SPICA}$ telescope allows mid-IR to submm observations which are limited only by the natural backgrounds, and BLISS is designed to operate near this fundamental limit. BLISS-SPICA provide a line sensitivity of $10^{-20} \mathrm{~W} \mathrm{~m}^{-2}$, thereby enabling spectroscopy of dust-obscured galaxies at all epochs back to the first billion years after the Big Bang (redshift $6)$, and study of all stages of planet formation in circumstellar disks.

BLISS covers the $35-430$ micron waveband at moderate resolving power $(300<\mathrm{R}<700)$ in six grating spectrometer bands, each coupling at least two 2 sky positions simultaneously. The instrument is cooled with an on-board refrigerator to $50 \mathrm{mK}$ for optimal sensitivity. The detector package in the goal implementation is 4200 silicon-nitride micro-mesh leg-isolated bolometers with superconducting transition-edge-sensed (TES) thermistors, read out with a cryogenic time-domain multiplexer. The instrument is designed to fit within the stringent SPICA resource allocations for mass and heat lift, and to mitigate the impact of cosmic rays. We report on this design and our progress in prototyping and validating the BLISS spectrometers and prototype cooler. A companion paper in Conference 8452 (A. Beyer et al.) discusses in greater detail the progress in the BLISS TES bolometer development.
\end{abstract}

Keywords: Bolometer, TES, Far-IR Spectroscopy

\section{INTRODUCTION}

The far-IR spectral regime is the repository of half the electromagnetic energy released in the history of stars and galaxies, and offers the only opportunity to probe the details of embedded star and planet formation and the processes in dust-obscured galaxies. Yet the far-IR has remained a relatively unexplored frontier because sensitive far-IR measurements require a combination that has yet to be fully realized: a large cryogenic telescope above the atmosphere and sensitive far-IR focal-plane technologies. The multinational SPICA team is now poised to provide provide this capability. The most important attribute of SPICA is its sub-6 K temperature. To understand this advantage, consider that at $\lambda=100 \mu \mathrm{m}$, even a well-designed passively-cooled $T \sim 80 \mathrm{~K}$ telescope such as Herschel is more than 10,000 times brighter than the natural astrophysical background due to solar-system and Galactic dust. Cooling the telescope to below $6 \mathrm{~K}$ virtually eliminates its emission relative to this background. The sensitivity improvement afforded by a cold telescope can be dramatic, as resoundingly demonstrated with Spitzer's successes in the mid- and far-infrared with only an $85-\mathrm{cm}$ aperture. SPICA is conceived in this same philosophy, and JAXA has invested significantly over the past decade to develop and verify closed-cycle 4-K and 2-K cryocoolers to enable a cryogen-free 5 -year mission.

Send correspondence to CMB: e-mail: bradford@caltech.edu, phone: 8183937499

Space Telescopes and Instrumentation 2012: Optical, Infrared, and Millimeter Wave, edited by Mark C. Clampin, Giovanni G. Fazio, Howard A. MacEwen, Jacobus M. Oschmann, Jr., Proc. of SPIE Vol. 8442, 84420P

(C) 2012 SPIE · CCC code: $0277-786 / 12 / \$ 18 \cdot$ doi: $10.1117 / 12.926843$

Proc. of SPIE Vol. $844284420 \mathrm{P}-1$ 


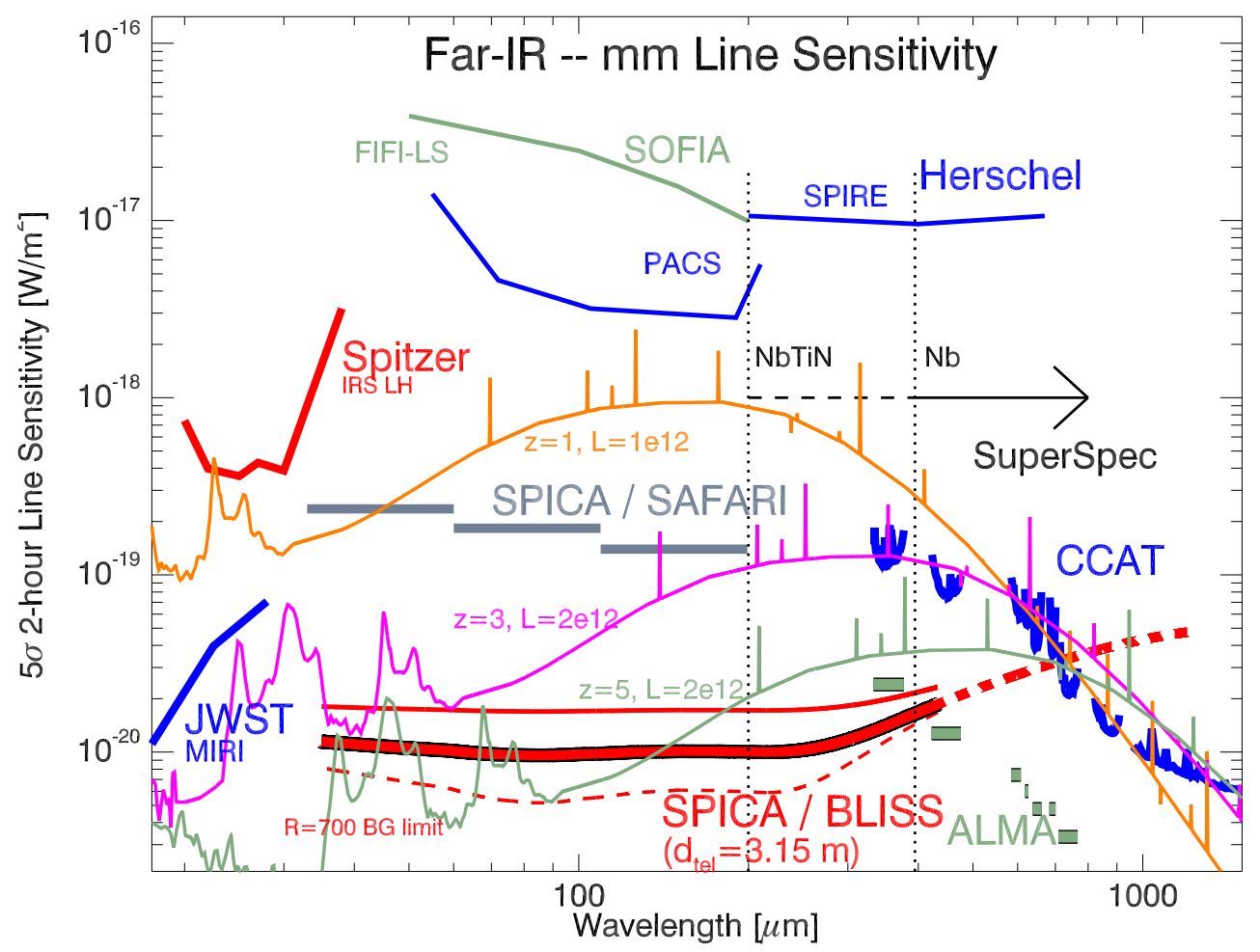

Figure 1. BLISS-SPICA is 100-1000 times more sensitive than present-day far-IR facilities for spectroscopy (and observing speed scales as the inverse square of the plotted sensitivity). BLISS sensitivities assume a 3.15-meter SPICA telescope at $5.5 \mathrm{~K}$ with $4 \%$ emissivity into the beam and $75 \%$ aperture efficiency. The spectrometers are assumed to be singlepolarization with a transmission of $25 \%$, including the detector quantum efficiency, and the sensitivites refers to an $\mathrm{R}=700$ bandwidth. Chopping the source between the two spectrometers is assumed, and an additional factor of two is included to account for additional losses and inefficiencies. The dashed curved at bottom is the photon background limit, the heavy curve above it assumes the BLISS detector goal sensitivity of $5 \times 10^{-20} \mathrm{~W} \mathrm{~Hz}^{-1 / 2}$. The lighter line above is calculated assuming the BLISS detector sensitivity requirement of $1 \times 10^{-19} \mathrm{~W} \mathrm{~Hz}^{-1 / 2}$. The SAFARI sensitivities are the goal values, taken from the SPICA yellow book, but adjusted to correspond to the 3.15-m aperture. The CCAT curves show sensitivities of spectrographs proposed for the Cerro-Chajnantor Atacama Telescope, a 25-meter submillimeter telescope planned for the high Atacama. Overplotted are redshifted galaxy spectra with $\mathrm{L}_{\text {tot }}=10^{12} \mathrm{~L}_{\odot}$ and assuming $\mathrm{L}_{\text {line }}=10^{9} \mathrm{~L}_{\odot}$ for the bright far-IR fine-structure transitions. 'SuperSpec' denotes the wavelength range accessible to a new on-chip wideband spectrometer using $\mathrm{Nb}$ and NbTiN superconductors under development at Caltech / JPL, presented in detail in conference 8452 by Shirokoff et al., Kovacs et al., and Barry et al.

\section{SCIENTIFIC PROMISE OF BLISS ON SPICA}

The sensitive SPICA platform is especially compelling for wideband spectroscopic follow-up, and BLISS is designed to provide this capability. Over SPICA's 5-year lifetime, BLISS will obtain full-band spectra of thousands of objects ranging from the first dusty galaxies to the most heavily enshrouded young stars and proto- planetary disks in our own Galaxy. These BLISS-SPICA spectra will directly address several pressing scientific topics, in particular:

2.1 The birth of planetary systems.

2.2 The history of cosmic star formation.

2.3 The connection between galaxies and supermassive black holes.

2.4 The onset of heavy elements and the rise of organic molecules in the Universe.

\subsection{Planetary-System Formation in the Milky Way: Gas in Disks}

The evolution of circumstellar disks and their gas component is key to planet formation. Disks rapidly evolve from the primordial gas-rich phase to planetary systems largely devoid of gas, placing stringent time constraints 

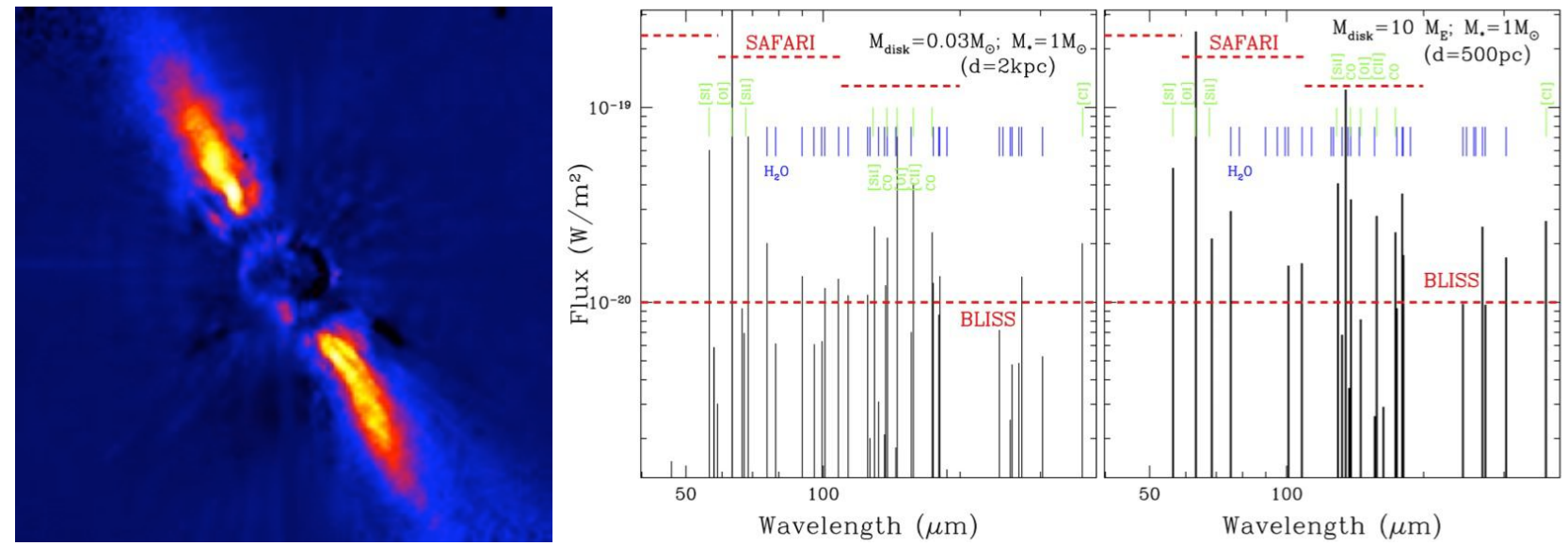

Figure 2. Diagnostics of planet-forming disks accessible with BLISS-SPICA. LEFT: Image of Beta Pic, a nearby (20 pc) disk system. CENTER: Model spectra of a primordial disk with $0.03 \mathrm{M}_{\odot}$ of gas at 2 kpc. The dust continuum is subtracted to show the line fluxes and compare with sensitivities. RIGHT: Model spectrum of an evolved debris disk where only 10 earth masses of gas remains, assuming $d=500$ pc. BLISS is required for detecting the faint emission lines from these such disks, and enables a comprehensive survey of gas in hundreds of star-forming disks of all ages. Model spectra provided by BLISS science co-I Uma Gorti (NASA Ames).

on the availability of gas for giant planet formation according to leading core accretion theories. ${ }^{1}$ Even small amounts of residual gas at late stages can affect the settling and radial drift of dust grains, planetary migration and eccentricity evolution. ${ }^{2-4}$ Spitzer mid-infrared spectroscopy has detected many atomic, ionic and molecular gas emission lines that arise from the inner 1-20 AU regions of disks, but the bulk of the disk mass is in the outer disk that emits primarily in the far-IR.

BLISS is ideal for studying gas in disks throughout the evolutionary sequence, from very young, primordial massive disks (with $\sim 0.01 \mathrm{M}_{\odot}$ ) to older, evolved disks with tiny amounts $\left(\sim\right.$ few $\mathrm{M}_{\text {Earth }}$ ) of remnant gas $($ see Figure 2). The various emission lines in the BLISS band originate from different regions of the disk and will trace the surface density distribution of disks as they evolve, form planets and eventually dissipate. The BLISS sensitivity is crucial for the full evolutionary census. Herschel/SPIRE is limited to the local (d<150 pc) starforming environments where only low-mass star formation occurs, and even SAFARI in SPICA will be unable to probe evolved low-gas-mass systems beyond a few hundred parsecs. The high sensitivity of BLISS will enable astronomers to detect gas emission from circumstellar disks around stars at kiloparsec distances, thus sampling many dense young clusters in giant molecular clouds, the dominant sites for low and high-mass star (and likely planet) formation.

\subsection{Charting the Cosmic History of Star Formation}

Far-IR and submillimeter continuum imaging surveys are now revealing cosmologically-significant populations of high-redshift galaxies which are so highly obscured that they emit nearly all of their energy in the mid-IR through submillimeter. These datasets, as well deep X-ray surveys show that much of the formative growth of stellar populations and black holes has been deeply obscured by dust for the bulk of the Universe's history. The high dust obscuration makes these galaxies inaccessible to astronomers' traditional diagnostic toolkit: rest-frame UV-optical spectroscopy. With its excellent spectral sensitivity in the $35-433 \mu m$ band, BLISS brings a powerful new toolkit to bear on these high-redshift galaxy populations: the rest-frame mid- to far-IR, where the dust becomes optically thin, and the dominant interstellar coolants lie (Figs.3,4). Each BLISS spectrum of a distant galaxy will:

- Immediately provide an unambiguous redshift, or look-back time for the galaxy.

- Determine the total star formation rate in the galaxy and infer a spatial scale of the buried starburst regions by comparing the intensities of the atomic gas coolants- $\mathrm{Si}^{+}, \mathrm{C}^{+}$, and $\mathrm{O}^{0}$ - with the total far-IR continuum intensity. In aggregate, these measurements chart the time history of dust-obscured stellar power output. 

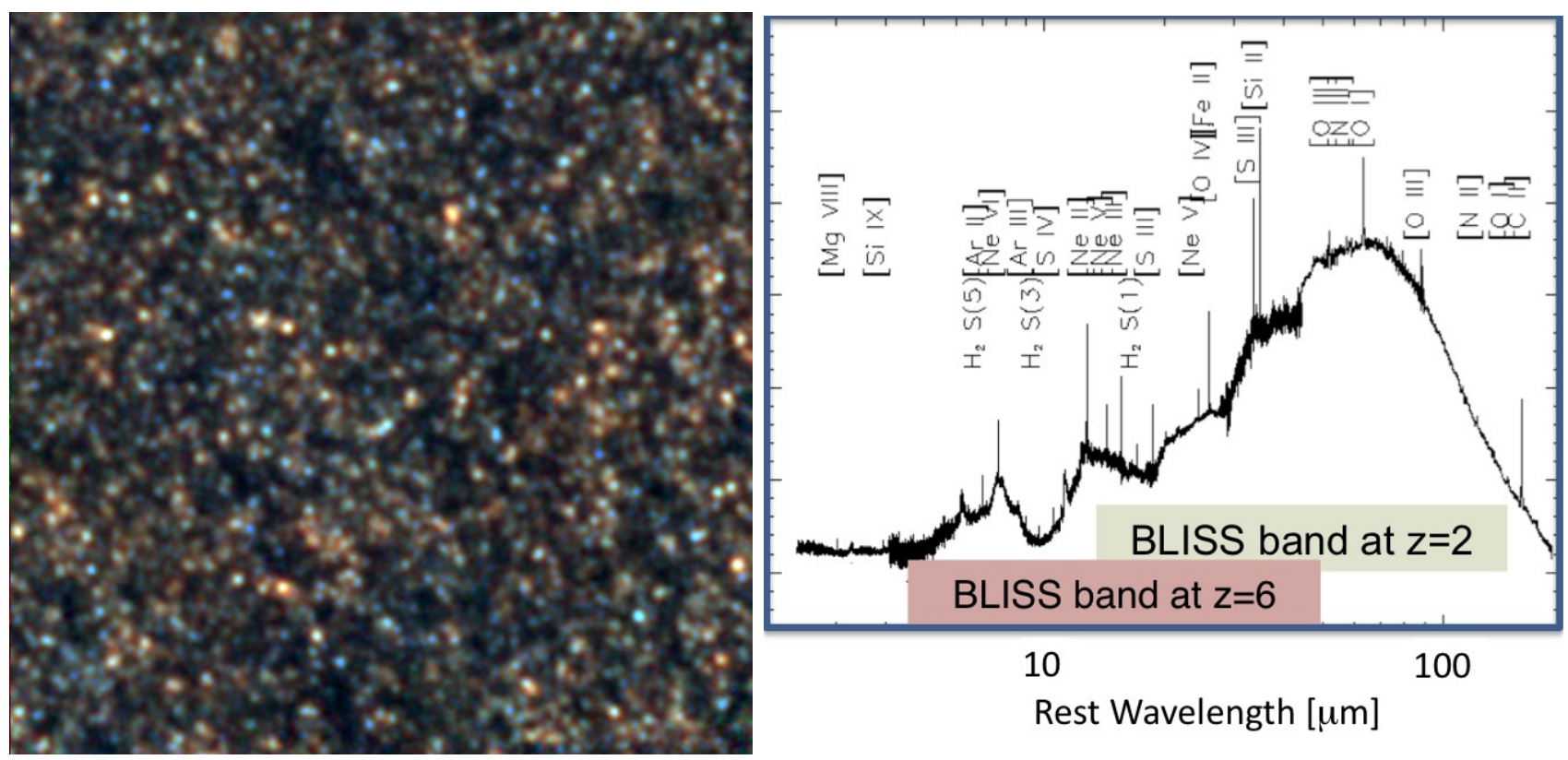

Figure 3. LEFT: Herschel/SPIRE 3-color image from the HerMES program. ${ }^{5}$ All points in the image are distant dusty galaxies. Most are likely at cosmological redshift $(\mathrm{z} \sim 1-5)$; their light originated in the first half of the Universe's history when star formation was much more vigorous than at present. The spectrometers on Herschel are not sensitive enough for spectroscopy of even the brightest of these galaxies. RIGHT: The Infrared Space Observatory (ISO) spectrum of the Circinus galaxy, a less-luminous nearby analogue of the high-z dusty galaxies. The spectral lines provide a redshift and probe the contents and energy sources deep within the dusty core. SPICA/BLISS will be capable of obtaining a similar spectrum for any of the galaxies identified in the image on the right. Over the life of SPICA, BLISS will obtain thousands of spectra like this one of galaxies at all ages.

- Measure the stellar initial mass function (IMF) via its effect on the UV field and the resulting ionization structure reflected in the fine-structure lines of ions: $\mathrm{O}^{++}, \mathrm{Ne}^{++}, \mathrm{N}^{++}, \mathrm{S}^{++}$, and $\mathrm{N}^{+}, \mathrm{Ne}^{+}$. By measuring the IMF verses time, BLISS will confirm or refute the hypothesis of a top-heavy IMF when the Universe was half its age, proposed to explain the discrepancy between stellar mass and energy release measurements.

\subsection{Revealing the Black-Hole-Galaxy Connection}

Accretion onto black holes (aka active galactic nuclei-AGN) is the second principal energy source in galaxies and it can be substantial. In the local-universe, ultraluminous infrared galaxies (ULIRGs) may derive up to $40 \%$ of their power from buried AGN, ${ }^{6,7}$ and stacking of faint $1<z<3$ ULIRGs selected with Spitzer at $24 \mu m$ also suggests a significant contribution from buried AGN to the total FIR background power. ${ }^{8,9}$ Moreover, there are indications that periods of significant black hole growth are associated with episodes of enhanced host-galaxy star formation, both from models and observations. ${ }^{10-12}$ This is intriguing, but spectroscopic confirmation is required to establish the presence of AGN, measure the partitioning of the energy between the AGN and star formation, and study the interaction between the AGN and the host galaxy. The same wideband far-IR spectra that probe the star-formation properties will also find and study buried AGN in the early Universe via:

- Direct measurement of the plasma around the accretion zone itself with fine-structure transitions of highionization-state species such as $\mathrm{Ne}^{4+}$ and $\mathrm{O}^{3+}$ (I.P. $\left.97 \& 54 \mathrm{eV}\right)$.

- Discovery of the warm $(\sim 1000 \mathrm{~K})$, dense $\left(10^{7} \mathrm{~cm}^{-3}\right)$ molecular torus believed to exist around the AGN-a likely waypoint as material is funneled from the host galaxy down to the accretion zone. It is expected to emit strongly in the high-J CO rotational transitions $\left(\lambda_{\text {rest }} \sim 50-80 \mu \mathrm{m}\right)$, easily detectable with BLISS to $\mathrm{z}=4$.

- Study of shocked $\mathrm{H}_{2}$ generated where AGN outflows impinge upon their host galaxies and the intergalactic medium. Such shocks cool via the $\mathrm{H}_{2}$ rotational transitions. 

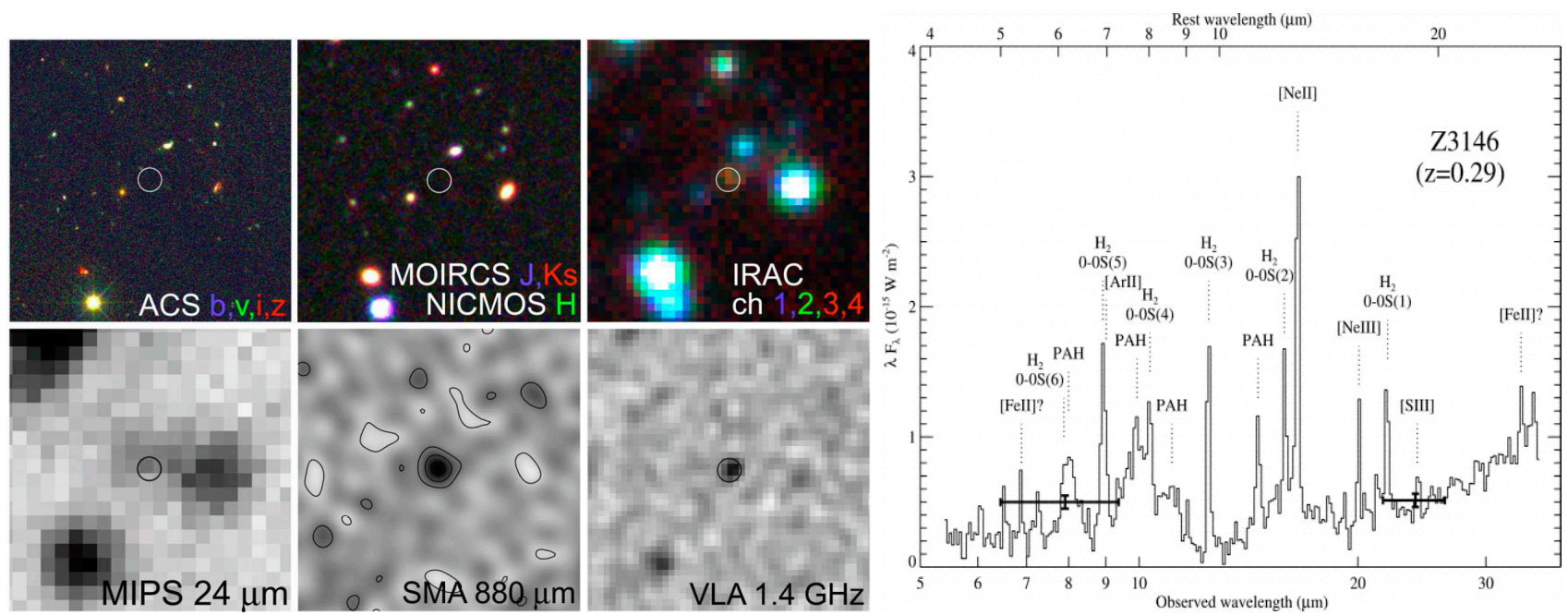

MIPS $24 \mu \mathrm{m}$
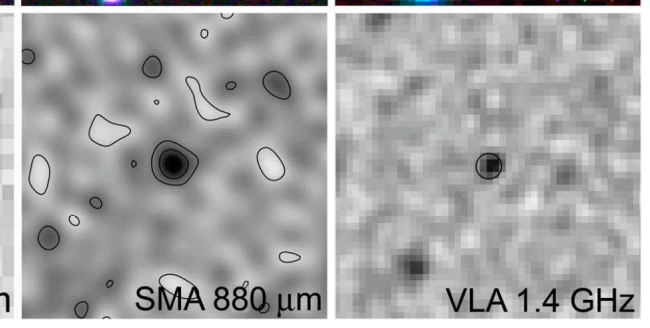

Figure 4. LEFT: Multiwavelength images $\left(24^{\prime \prime}\right.$ on a side) toward a submillimeter-bright galaxy discovered with Scuba/JCMT in the GOODS-N field and confirmed using the SMA submillimeter interferometer (center bottom) which provides an accurate position (from Wang et al. 2009 ${ }^{13}$ ). The object is undetected in deep HST/ACS, HST/NICMOS, and Subaru/MOIRCS images, and is very faint in the Spitzer IRAC, Spitzer MIPS-24 $\mu$ m, and VLA images. The redshift is unknown but thought to be $z \sim 4-6$. RIGHT: Spitzer IRS spectrum of the Zw 3146 from Egami et al. (2006) ${ }^{14}$ showing bright $\mathrm{H}_{2}$ and PAH emission. These lines and PAH features would be detectable with BLISS - SPICA at $\mathrm{z}=8-10$.

\subsection{Charting the Rise of Heavy Elements and Organic Molecules}

As the Universe is enriched from primordial $\mathrm{H}_{2}$ to a medium which contains heavy elements and dust grains, the key cooling pathways shift from the quadruple pure rotational $\mathrm{H}_{2}$ lines $(28,17,12,9.7,8.0,6.9 \ldots \mu m)$ to a combination of the fine-structure transitions discussed above and the dust. BLISS will probe all phases of this transition. For metallicity above $\sim 10^{-4}$ solar, fine-structure lines are believed dominate $\mathrm{H}_{2}$ cooling. However, surprisingly powerful $\mathrm{H}_{2}$ emitters (e.g., Stephan's Quintet, Zw3146) have been found at low-redshift with Spitzer. ${ }^{15,16}$ These nearby sources may be analogs of early-Universe shocks produced in galaxy formation and AGN feedback, when dust and metals are emerging from the first cycles of enrichment. For $z \sim 5-10$, the $\mathrm{H}_{2}$ lines are redshifted into the far-IR, and remarkably, Zw3146 itself would be detectable in its $\mathrm{H}_{2}$ lines with $B L I S S-S P I C A$ even at $z \sim 8-10$.

Once heavy elements are in place, the rest-frame mid-IR dust features may actually be the most practical probe of heavy elements at early times. Dust is believed to form as the first heavy elements are created, for example in pair-instability Population III supernovae remnants, and Spitzer has shown that the dust features are often the most powerful features in the spectra of galaxies at all wavelengths. In particular, the polycyclic aromatic hydrocarbon $(\mathrm{PAH})$ features at $6.2-17 \mu \mathrm{m}$ are unambiguous, with to $15 \times$ more power than the brightest atomic cooling lines, and act as sensitive probes of heavy element abundance. Like the $\mathrm{H}_{2}$ lines, they are redshifted out of the JWST band, but not into the ALMA windows in the $\mathrm{z} \sim 5-10$ era. BLISS can detect these powerful bands at early epochs (Fig. 1), thus probing the transition from primordial $\mathrm{H}_{2}$ to heavy-element cooling in the billion years of the Universe.

The excellent sensitivity of BLISS is essential for these distant-galaxy measurements. Charting a complete history requires study of galaxies before, during, and since the putative era of peak star formation and black hole growth between 2-6 billion years after the Big Bang. To reach the first billion of the Universe $(\mathrm{z}=6)$ in the spectral probes demands a line sensitivity of $10^{-20} \mathrm{~W} \mathrm{~m}^{-2}$, which is only achieved with an actively-cooled telescope and a sensitive grating spectrometer such as BLISS. We note that a potential concern for probing faint objects from the very early epochs (e.g. $\mathrm{z}=6$ ) is confusion in the spectrum created by intervening galaxies (e.g. z 1-3) which lie within the beam along the line of sight. We are assessing this numerically by combining far-IR galaxy models with model spectra and are preparing an article for the ApJ (E.J. Murphy et al.). Our preliminary finding is that the density of lines at the BLISS detection threshold per beam and per $\mathrm{R}=700$ bin in an average sightline is $\sim 10^{-2}$ (depending on wavelength). Thus, while multiple sources may contribute to a 
Table 1. BLISS Specifications, Key Technologies, and Heritage

\begin{tabular}{|c|c|c|c|c|}
\hline Parameter & Goal & Requirement & \multicolumn{2}{|l|}{ Driver } \\
\hline $\begin{array}{l}\text { Line sens. }(5 \sigma, 1 \mathrm{~h}) \\
\text { Resolving power } \\
\text { Spectral coverage } \\
\text { Number of beams }\end{array}$ & $\begin{array}{l}1 \times 10^{-20} \mathrm{~W} \mathrm{~m}^{-2} \\
700 \\
2(\text { sourc }\end{array}$ & $\begin{array}{l}2 \times 10^{-20} \mathrm{~W} \mathrm{~m}^{-2} \\
300 \\
\mu m \\
\text { reference) }\end{array}$ & \multicolumn{2}{|c|}{$\begin{array}{l}\text { Galaxies, systems at cosmic dawn } \\
\text { Avoids line confusion in deep obs. } \\
\text { Full coverage w/ JWST, ALMA } \\
\text { Redundancy for single pixel failure }\end{array}$} \\
\hline $\begin{array}{l}\text { Detector format } \\
\text { Detector sensitivity } \\
\text { Detector time const }\end{array}$ & $\begin{array}{l}4224 \\
5 \times 10^{-20} \mathrm{~W} / \sqrt{\mathrm{Hz}} \\
<80 \mathrm{~ms}\end{array}$ & $\begin{array}{l}2112 \\
1 \times 10^{-19} \mathrm{~W} / \sqrt{\mathrm{Hz}} \\
<160 \mathrm{~ms}\end{array}$ & $\begin{array}{l}2 \text { beams, full spec coverage } \\
\text { Supports instrument sensitivi } \\
\text { Modulate at } \mathrm{f}>0.8 \mathrm{~Hz}\end{array}$ & \\
\hline Subsystem & \multicolumn{2}{|l|}{ Approach } & Heritage & TRL \\
\hline $\begin{array}{l}\text { TES bolometer system } \\
\text { Low-NEP detector } \\
\text { Detector cold readout } \\
\text { Warm electronics }\end{array}$ & $\begin{array}{r}\text { JPL TI } \\
65 \mathrm{mK} \mathrm{MoCu} \\
\text { NIST time-do } \\
\text { Multi-channel }\end{array}$ & $\begin{array}{l}\text { olometer } \\
\text { on nitride mesh } \\
\text { ctronics (MCE) }\end{array}$ & $\begin{array}{l}\text { BICEP2, SPIDER } \\
\text { JPL development program } \\
\text { BICEP2, SPIDER, SCUBA2 } \\
\text {...same... }\end{array}$ & $\begin{array}{l}5 \\
4 \\
5 \\
5\end{array}$ \\
\hline $\begin{array}{l}\text { Short- } \lambda \text { spectrometer } \\
\text { Long- } \lambda \text { spectrometer }\end{array}$ & \multicolumn{2}{|c|}{$\begin{array}{l}\text { Cross-dispersed echelle grating } \\
\text { WaFIRS waveguide grating }\end{array}$} & $\begin{array}{l}\text { Spitzer IRS } \\
\text { Z-Spec }\end{array}$ & $\begin{array}{l}6 \\
5\end{array}$ \\
\hline $\begin{array}{l}\text { Instrument cooler } \\
\text { Intercept cooler }\end{array}$ & \multicolumn{2}{|c|}{$\begin{array}{c}50 \mathrm{mK} \text { adiabatic demag ADR } \\
300 \mathrm{mK} \text { continuous }{ }^{3} \mathrm{He} \text { sorption }\end{array}$} & $\begin{array}{l}\text { Z-Spec (Astro-E2) } \\
\text { Herschel SPIRE }\end{array}$ & $\begin{array}{l}6 \\
8\end{array}$ \\
\hline
\end{tabular}

composite spectrum, line confusion should not be a problem at this density.

\section{BLISS TECHNICAL APPROACH}

The scientific objectives require a line sensitivity approaching $10^{-20} \mathrm{~W} \mathrm{~m}^{-2}$, large instantaneous bandwidth, and moderate spectral resolving power $(R=\lambda / \delta \lambda \geq 300)$. The combination of sensitivity and bandwidth is only possible with a grating spectrometer, and the detectors must have sensitivity which is close to the fundamental photon background noise of the zodiacal and Galactic dust. The BLISS wavelength range fills the gap between the 35- $\mu \mathrm{m}$ cutoff of the doped Si BIB arrays (planned for the mid-IR instruments), and the short submillimeter, where observations from the ground with ALMA and single-dish telescopes such as CCAT become practical. These requirements and the key design choices for BLISS are presented in Table 1.

BLISS mounts to the T 4.5 K SPICA instrument optical bench (IOB), viewing an off-axis field through a pickoff mirror. A 3-mirror relay reimages the SPICA focal plane for BLISS; the 2nd relay mirror is at an image of the primary and is chopped to provide beam-switching modulation which is optimal for the bolometers. The relay design has flexibility, and can accommodate a range of positions for BLISS within the IOB, depending on the needs of the other instruments. Thermally, BLISS rejects heat to the SPICA $1.7-\mathrm{K}$ and $4.5-\mathrm{K}$ heat sinks to cool the spectrometers and detectors to $50 \mathrm{mK}$ as well as a thermal intercept stage.

Table 2. BLISS Bands and Bolometer Parameters (Goal System)

\begin{tabular}{|c|c|c|c|c|c|c|c|c|c|c|c|c|}
\hline Bd. & $\begin{array}{l}\lambda_{\min } \\
{[\mu \mathrm{m}]} \\
\end{array}$ & $\begin{array}{l}\lambda_{\max } \\
{[\mu \mathrm{m}]}\end{array}$ & $\begin{array}{l}\text { Det s } \\
\text { spat }\end{array}$ & $\begin{array}{l}{[\mu \mathrm{m}]} \\
\mathrm{spec}\end{array}$ & $\begin{array}{c}\mathrm{Q} \\
{[\mathrm{aW}]} \\
\end{array}$ & $\begin{array}{l}\text { NEP } \\
\text { Phot }\end{array}$ & $\begin{array}{l}\overline{-20} \mathrm{~T} \\
\text { Det }\end{array}$ & $\begin{array}{l}/ \sqrt{\mathrm{Hz}}] \\
\mathrm{Mrgn}\end{array}$ & $\begin{array}{c}\mathrm{G} \\
{[\mathrm{fW} / \mathrm{K}]}\end{array}$ & $\begin{array}{c}\tau \\
{[\mathrm{ms}]}\end{array}$ & $\begin{array}{c}\text { Dyn } \\
\mathrm{MoCu} \\
\end{array}$ & $\begin{array}{c}\text { range } \\
\mathrm{Ti} \\
\end{array}$ \\
\hline \multicolumn{13}{|c|}{ Cross-Dispersed Echelle Modules } \\
\hline 1 & 34.5 & 52.6 & 1380 & 430 & 0.12 & 3.1 & 2.8 & 5.0 & 4 & 80 & 400 & 15000 \\
\hline 2 & 52.6 & 80.2 & 1380 & 430 & 0.12 & 2.8 & 2.8 & 5.0 & 4 & 80 & 400 & 15000 \\
\hline 3 & 80.2 & 122 & 2100 & 650 & 0.13 & 2.4 & 2.8 & 5.0 & 4 & 80 & 400 & 15000 \\
\hline \multicolumn{13}{|c|}{ Waveguide Far-IR Spectrometer (WaFIRS) Modules } \\
\hline 4 & 122 & 186 & 873 & 140 & 0.25 & 2.6 & 2.8 & 5.0 & 4 & 80 & 200 & 8000 \\
\hline 5 & 186 & 284 & 1350 & 216 & 0.34 & 2.4 & 2.8 & 5.0 & 4 & 120 & 150 & 6000 \\
\hline 6 & 284 & 433 & 2100 & 336 & 2.3 & 5.0 & 5.0 & 5.0 & 12 & 150 & 75 & 3000 \\
\hline
\end{tabular}

Notes: NEP columns are photon noise, design detector NEP, and detector NEP including margin. Detector noise includes all sources of detector and readout noise with an operating impedance of $10 \mathrm{mOhms}$ with $2 \mathrm{pA} / \sqrt{\mathrm{Hz}}$ unmultiplexed SQUID noise with TES transitions at $65 \mathrm{mK}(\mathrm{MoCu})$ and $450 \mathrm{mK}(\mathrm{Ti})$. 


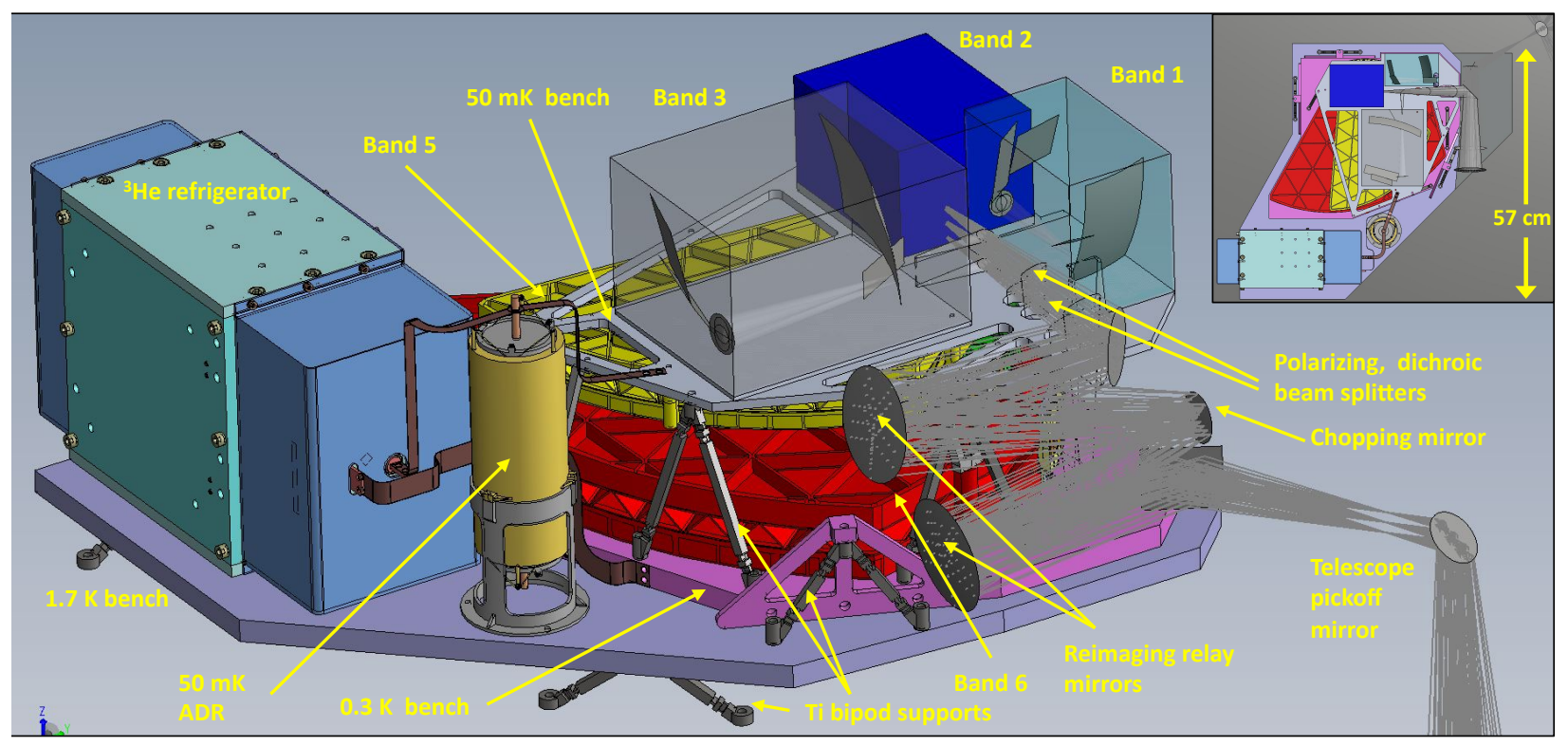

Figure 5. BLISS conceptual layout. Spectrometer modules are colored by band. Band 4 is between Bands 5 and 6 , not visible in this view. Radiation shields are not shown, but will be included at the 1.7 and $0.3 \mathrm{~K}$ stages. This layout includes Band 6 sized for $\mathrm{R}=700$; it will likely be reduced to meet available allocations.

\subsection{A Multi-Band Grating Spectrometer}

\subsection{BLISS System Approach}

The 38-433 $\mu \mathrm{m}$ BLISS range is covered in six bands (Table 2), each with its own wideband spectrometer module (Figs. 5,6). We are targeting a resolving power $R$ of 700, but note that for the longest-wavelength band the resolution may have to be reduced since it drives the volume and mass. The goal is that all bands will couple the same positions on the sky through the use of a polarizer and dichroic filters. After a polarizing splitter, one linear polarization will couple bands 1, 3,5 and the other will couple bands 2, 4, 6, easing the requirement on the transition region for the dichroic filters. In this scenario, each spectrometer is only single-polarization, which is a natural property of the long-wavelength spectrometers anyway. The spectrometer bands will each couple two sky positions simultaneously, separated by $2-3$ arcminutes ( $4-6$ beams at the longest wavelength). The light from the astronomical source will be exchanged between these two spectrometer output positions with the chopping mirror operating at a single fixed frequency between 0.5 and $5 \mathrm{~Hz}$. The dual-field capability provides an important redundancy against loss of a spectral channel in the event of a single detector failing in one field. This is critical for BLISS since without moving parts inside the instrument there is no way to modulate the spectral response with respect to the array, and the spectral sampling is likely to be sub-Nyquist. The dual-field capability also doubles the observing speed.

BLISS will employ two types of broadband grating. At the long-wavelengths, conventional free-space grating designs would be too large and massive. BLISS will use the WaFIRS spectrometer architecture: a curved custom-ruled grating in parallel-plate waveguide coupled with a single-mode feed, ${ }^{17}$ which offers compact size and small mass. For the short-wavelength BLISS bands, waveguide devices are not an option as they require sub-wavelength manufacturing tolerances, here BLISS will employ conventional cross-dispersed echelle grating modules, similar to those used for the high-resolution modules of the Spitzer infrared spectrograph (IRS), but with a new maximally-compact design. We describe our progress in design and prototyping of both types of spectrometer modules.

\subsubsection{Cross-Dispersed Echelle Grating Design}

We are studying the design of the echelle grating modules and have developed a 'dual-field' approach, in which a common set of optics couples the light from both separate BLISS fields, and provides sufficient separation in the focal plane to accommodate the quasi-1D bolometer arrays. This enables the dual-field BLISS without the 


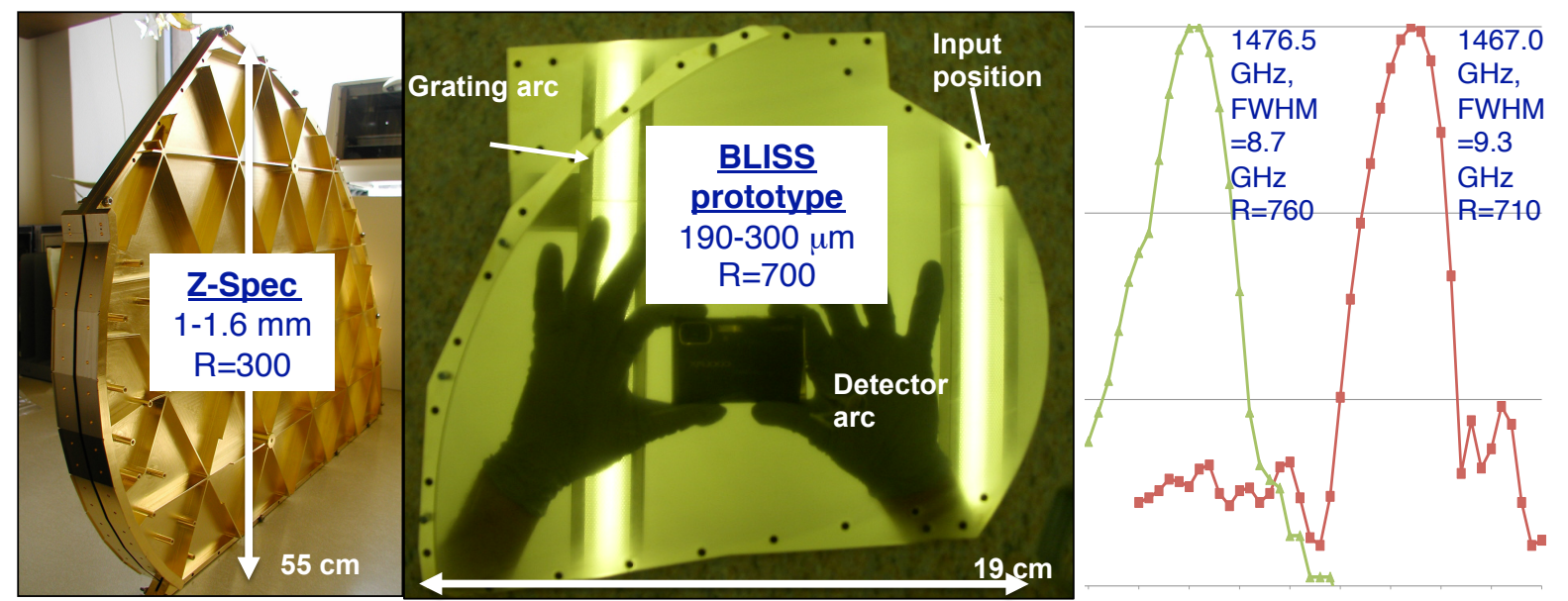

Figure 6. BLISS waveguide spectrometer demonstration. LEFT is the spectrometer with 481 grating facets used in the Z-Spec instrument. It provides $\mathrm{R}=300$ across the $1 \mathrm{~mm}$ atmospheric window (0.95-1.65 mm). Center is the BLISS prototype device with a 981-facet grating for 182-303 $\mu \mathrm{m}$ fabricated in the JPL shop (the top plate is removed). Right shows measured spectral profiles of this grating at two nearby frequencies using a local oscillator source and a scanned output feed coupling a $4 \mathrm{~K}$ bolometer. The horizontal axis is position of the on the output arc (total range of $2 \mathrm{~mm}$ in the plot), and the vertical axis is logarithmic with horizontal lines indicating orders of magnitude. The mapping of frequency to output position, and the resolving power $\left(\nu / \delta \nu_{\mathrm{FWHM}}\right)$ of the system are as designed.

second set of echelle spectrometers, easing the pressure on the mass budget. This approach favors a slightly smaller fractional bandwidth than used in the Spitzer IRS, and for BLISS the 35-120 $\mu \mathrm{m}$ range will be covered with three bands each with one of these dual-field modules.

\subsubsection{WaFIRS Spectrometer Development}

The WaFIRS architecture has been demonstrated on the Z-Spec instrument on Mauna Kea, ${ }^{18}$ but relative to the Z-Spec module (1-1.6 $\mathrm{mm}$ band at $R=250-350)$ both higher frequencies and higher resolving powers are required for BLISS. To demonstrate this basic capability of the WaFIRS architecture, we have built and begun testing a prototype for the 990 to $1650 \mathrm{GHz}$ band $(182-303 \mu \mathrm{m})$ with 981 facets in order to provide $R \sim 700$. Our initial measurements show that this device indeed provides the design resolving power, as shown in Figure 6. The WaFIRS spectrometers are an important term in the mass budget, and we have incorporated sufficient mechanical design and analysis to insure that structures can be built within the $30 \mathrm{~kg}$ mass budget which can survive launch loads for all the bands.

\subsection{Transition-Edge-Sensor (TES) Bolometers}

At present, the most viable approach for meeting the BLISS requirements is to use superconducting TES (transition-edge sensed) bolometer arrays with a SQUID (Superconducting QUantum Interference Device) timedomain multiplexing readout (MUX). The detector is a silicon nitride micro-mesh ('spider-web') bolometer patterned into a metalized grid absorber suspended by thin support legs, with a voltage-biased TES film as the thermistor. BLISS bolometer parameters are summarized in Table 2. Our goal is to produce devices which match the total detector noise, dominated by phonon (thermal fluctuation) noise $\mathrm{NEP}_{\text {phonon }} \simeq \gamma \sqrt{4 \mathrm{kT}} \mathrm{G}^{2}$, to the photon noise. This determines the thermal conductivity $\mathrm{G}$ for a $65-\mathrm{mK}$ TES operating from a $50 \mathrm{mK}$ base temperature. Lower $\mathrm{G}$ devices reducing the detector noise might be considered, but this provides diminishing improvement in system sensitivity, while impacting dynamic range to saturation optical power and response time which will be important for cosmic ray event removal. The goal instrument sensitivities are based on a detector NEP $=5 \times 10^{-20} \mathrm{~W} \mathrm{~Hz}^{-1 / 2}$, but we are actually targeting a somewhat lower $3 \times 10^{-20} \mathrm{~W} \mathrm{~Hz}^{-1 / 2}$ for the phonon noise itself, thereby providing additional margin for noise sources associated with imperfect instrument interfaces (e.g. EMI/EMC, straylight, etc). We set the detector NEP requirement at $1 \times 10^{-19} \mathrm{~W} \mathrm{~Hz}^{-1 / 2}$, which still provides excellent sensitivity. To enable both the faint-source sensitivity and adequate dynamic range for 


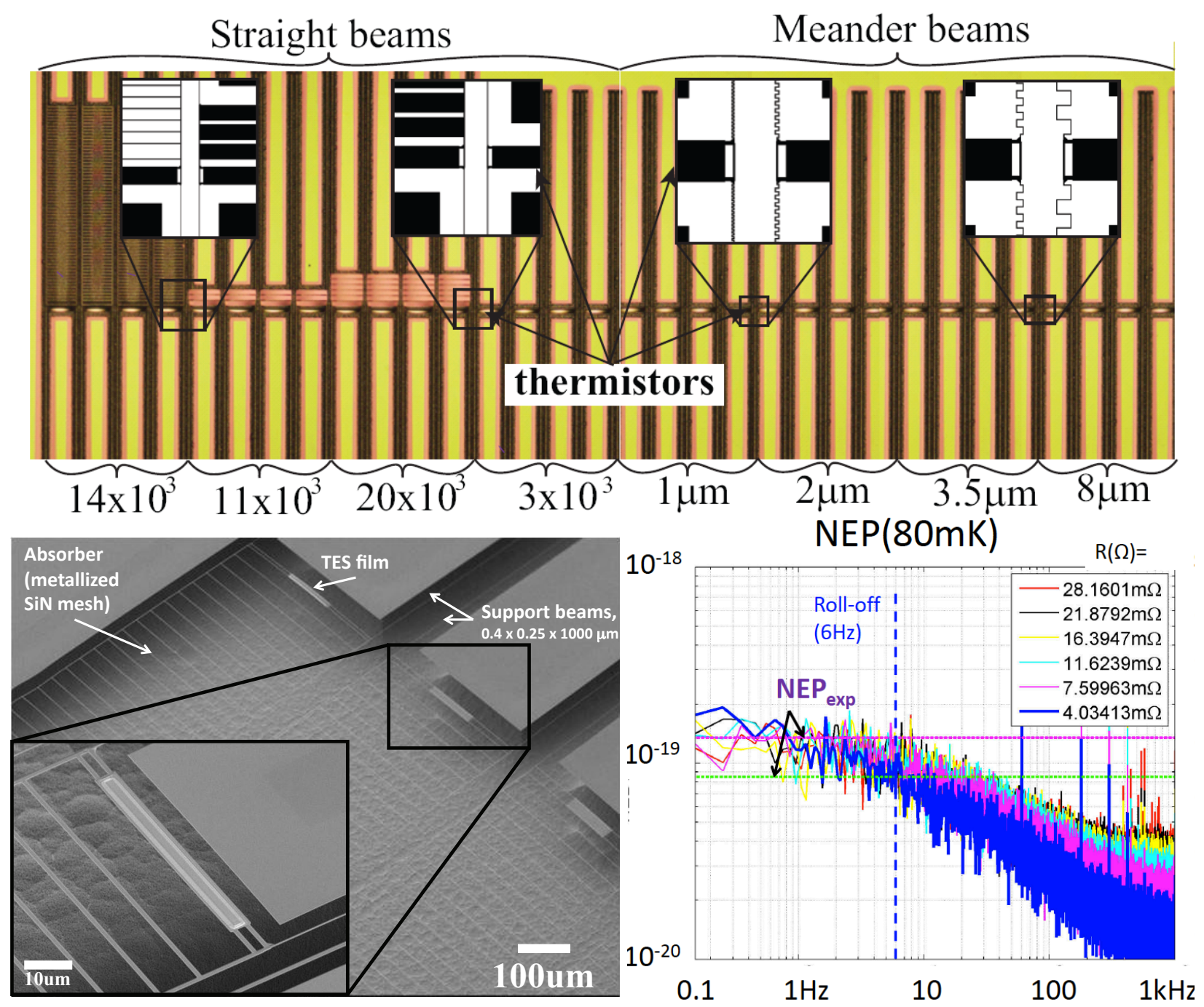

Figure 7. TOP: Prototype 32-element BLISS detector array showing a variety of absorber and isolation beam approaches. The 16 devices on the left each have 4 silicon nitride support beams $1 \mathrm{~mm}$ long $\times 0.4 \mu m$ wide $\times 0.25 \mu m$ thick. These 'straight-leg' pixels also have four absorber geometries with their area shown in $\mu m^{2}$ underneath the pixels. The long 'ladder' absorbers of the four left most pixels are the baseline design for BLISS. The other absorber geometries provide a range in nitride area to explore the nitride heat capacity. The right 16 pixels have meandered support beams with meanders constructed of straight segments of $1 \mu \mathrm{m}, 2 \mu \mathrm{m}, 3.5 \mu \mathrm{m}$ and $8 \mu \mathrm{m}$ in length, respectively (all with absorber area of $3000 \mathrm{\mu m}^{2}$ ). The insets show close-up views of portions of the geometry of the nitride for the eight types of pixels. This prototype array uses elemental iridium (Ir) for the thermistors, for which $\mathrm{T}_{\mathrm{C}}=130 \pm 20 \mathrm{mK}$. BOT, LEFT: Micrographs of BLISS prototypes. The large image shows 3 devices sized for Band 5 (with slightly different TES thermistor geometries). The inset shows a close up of a device sized for Band 3. The absorber will be patterned with gold and couples light polarized along the rungs of the ladder structure, as is required for the waveguide spectrometers. BOT, RIGHT: Example NEP from the above prototype array - this measurement refers to a meander-beam device with an Iridium thermistor. Expected NEPs are shown (not including any excess photon noise), the range results from the degeneracy between $\mathrm{T}_{\mathrm{c}}$ and the residual or 'dark' power inferred from load curves.

Galactic observations, the TES is a dual design using $\mathrm{MoCu}$ for low-background observations, in series with elemental Ti for high-background observations.

As we improve the fidelity of the our testbed to enable the $65 \mathrm{mK}$ devices, we are currently testing devices with elemental iridium thermistors, with Tc between 135-160 mK. These devices have yielded electrical noise measurements close to the BLISS requirement, as shown in Figure 7. Measurements of MoCu devices with 


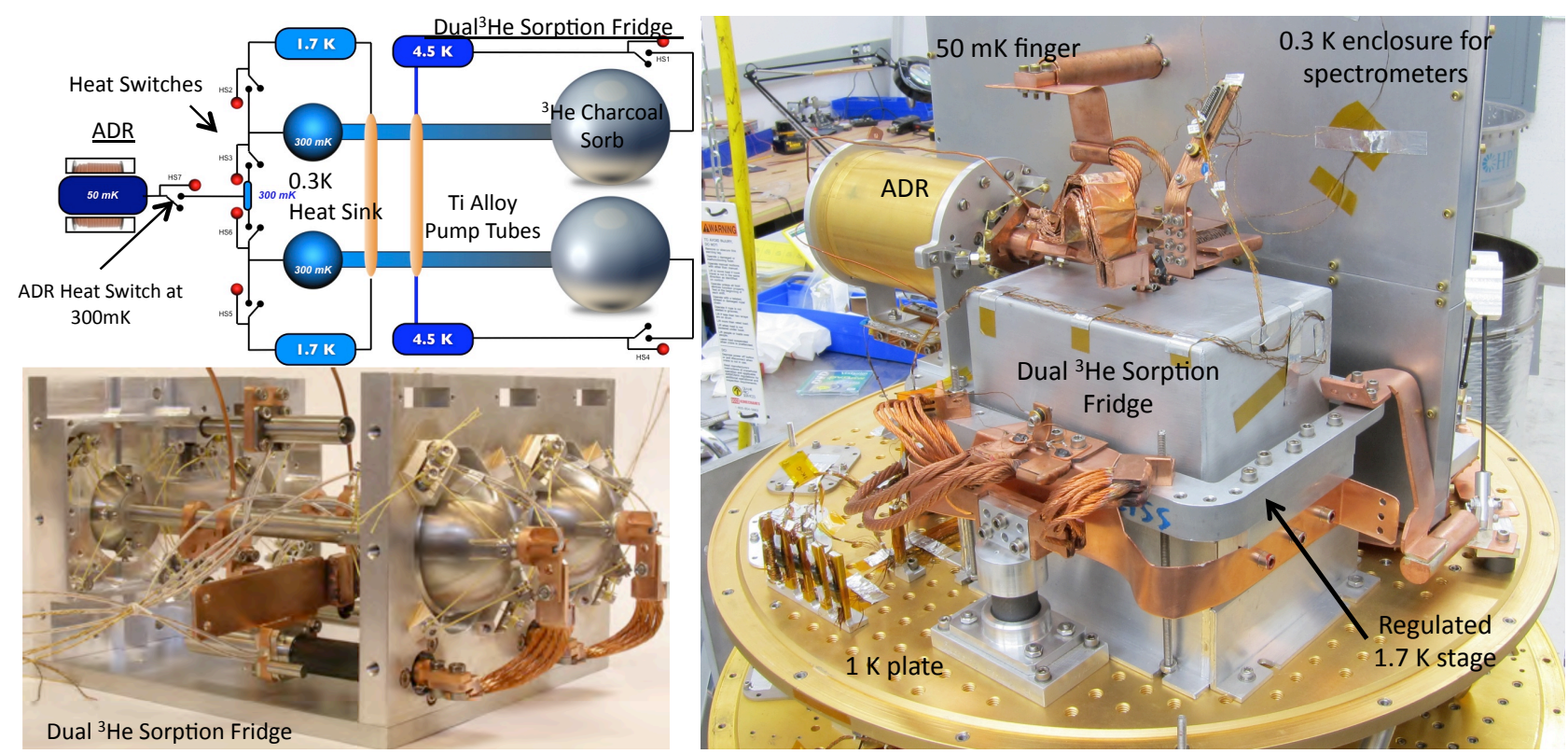

Figure 8. BLISS custom cooler prototype. Top, left shows the sub-K architecture: a dual ${ }^{3}$ He system provides continuous cooling at $0.3 \mathrm{~K}$, and a single-shot ADR cools the instrument to $0.05 \mathrm{~K}$. Bottom, left shows the continuous ${ }^{3} \mathrm{He}$ system procured from Lionel Duband (CEA Grenoble). Right shows integration with our ADR into a closed-cycle testbed which provides regulated platforms at $4.5 \mathrm{~K}$ and $1.7 \mathrm{~K}$, simulating the heat SPICA heat lift stages. The large $0.3-\mathrm{K}$ cooled enclosure surrounding a $50 \mathrm{mK}$ suspension will house the BLISS spectrometer modules for optical testing.

$\mathrm{T}_{\mathrm{c}} \sim 65 \mathrm{mK}$ are now underway. The progress with the BLISS bolometer development is presented in greater detail by A. Beyer et al. in SPIE Proceedings Volume 8452 and in Beyer et. al., 2010. ${ }^{19}$

\subsection{Cold Multiplexer and Warm Readout Electronics}

SQUID-based time-domain multiplexed (TDM) readouts developed at NIST Boulder are currently able to multiplex up to 40 detectors at the low-temperature stage, introducing negligible amplifier noise, and these readouts are being employed in numerous ground-based and sub-orbital experiments. At NIST we are developing a SQUID time domain MUX (TDM) suited to the BLISS requirements. The readout will use the new gangable 11-way linear MUX architecture which is an improvement over the standard 32-way system, ${ }^{20}$ and we have a low-power implementation specifically tailored for BLISS which uses a reduced critical current of the SQUID switches and an increased SQUID input inductance and shunt resistance. As with all modern TDM designs, the SQUID inductors are gradiometer wound to reduce magnetic field sensitivity. We have built and measured an 11-way chip which, when ganged in a group of 3, reads out 32 detectors (and 1 dark squid) and dissipates only 0.5 $\mathrm{nW}$, makign the MUX a negligible term in the $50 \mathrm{mK}$ thermal budget. The chips will interface to the detectors though a superconducting RF filter to protect the detectors from RF power, and Nyquist inductors tailored to the BLISS detector speed.

The warm readout and control electronics are derived from the proven systems developed for TDM SQUID focal planes and adapted for space. The BLISS readout will operate at a slower frame rate of $2 \mathrm{kHz}$, appropriate for the relatively slow detector speed, compared with the $15-20 \mathrm{kHz}$ rate used for ground-based systems. Furthermore, since the maximum saturation current on the BLISS detectors is much smaller than the periodic current on the input SQUIDs, the feedback control on the SQUIDs can be simplified, reducing the speed requirements on the DACs. These simplifications permit a square-wave bias on the detector, providing noise stability and reducing magnetic field susceptibility in the SQUID readouts.

\subsection{BLISS Cryogenic Architecture and Thermal Resource Requirements}

We have developed a complete thermal model of BLISS including masses based on optical designs, and a support structure sufficiently stiff to survive launch loads. The focal-plane modules are cooled to $50 \mathrm{mK}$ with a custom 

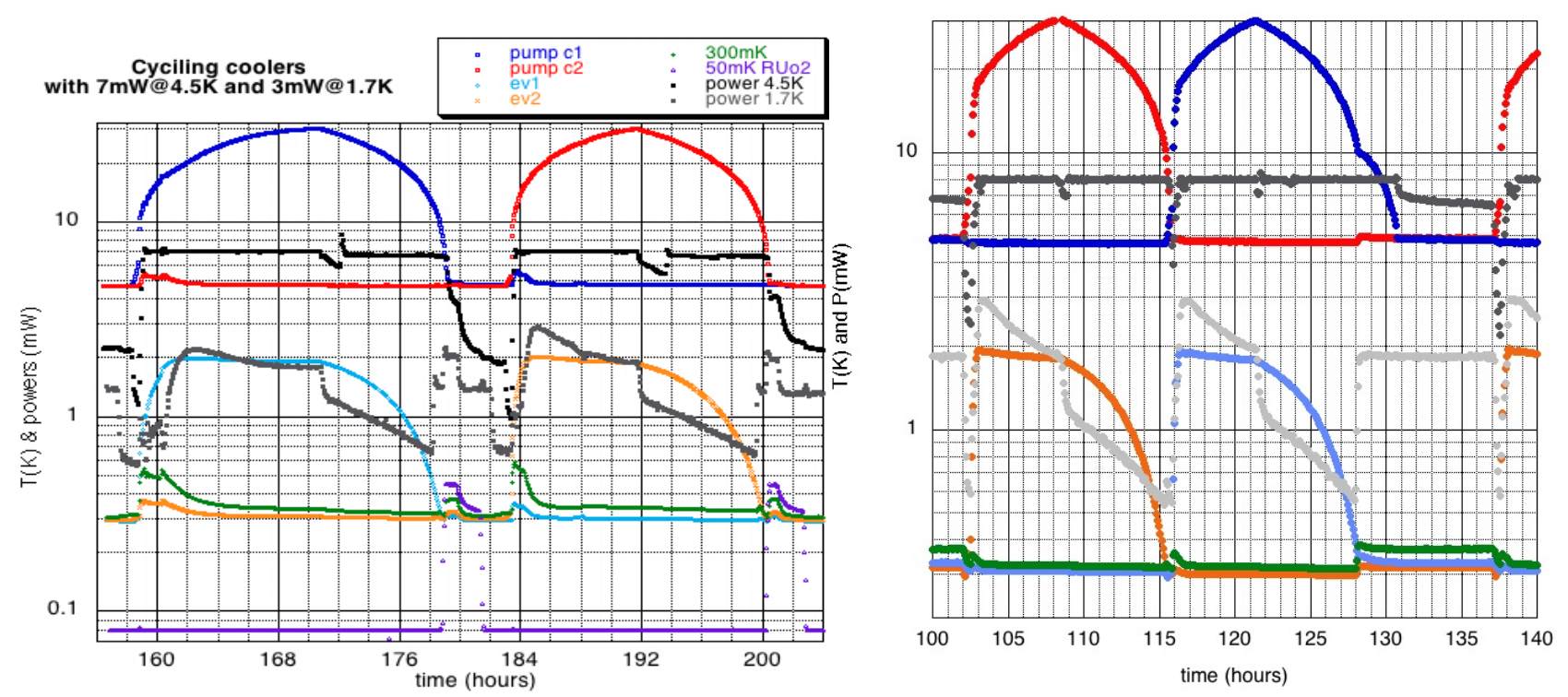

Figure 9. Operation of prototype BLISS cooler interfacing with $4.5 \mathrm{~K}$ and $1.7 \mathrm{~K}$ regulated cooling stages which mimic the SPICA high lift points. The two ${ }^{3} \mathrm{He}$ sorption coolers are cycled sequentially, for each the cycle is regulated to only reject $7 \mathrm{~mW}$ to the $4.5 \mathrm{~K}$ stage (black curve). The power rejected to $1.7 \mathrm{~K}$ (grey curve) is always under $3 \mathrm{~mW}$, typically less than $2 \mathrm{~mW}$. The green curve is the $300 \mathrm{mK}$ intercept stage temperature. The measurements plotted at left include the ADR stage, the glitches at the shifting between the sorption coolers are due to a slow heat switch response which has been eliminated. The ADR salt pill used thus far is not optimized for $50 \mathrm{mK}$ operation; we are developing a new pill to support the $50 \mathrm{mK}$ requirement. At right shows a more recent measurement without the ADR stage but with the new heat switches. The heat load from cycling the ADR is simulated with an applied load totaling 3.3 Joules between 129 and 136 hours.

dual-stage cooler that rejects heat to SPICA's $1.7 \mathrm{~K}$ and $4.5 \mathrm{~K}$ cold fingers, shown in Figure 8. A continuous intercept at $300 \mathrm{mK}$ is provided by two Herschel-style closed-cycle pumped- ${ }^{3} \mathrm{He}$ coolers ${ }^{21}$ such as those from INAC, France (Dr. Lionel Duband). These refrigerators are cycled sequentially on a $\sim 24$-hour interval and switched to the load with gas-gap heat switches. The detectors and spectrometers are cooled from $300 \mathrm{mK}$ to $50 \mathrm{mK}$ using a single shot adiabatic demagnetization refrigerator (ADR), similar to that used in Z-Spec ${ }^{18}$ and flown on Astro-E. ${ }^{22}$ The ADR is sized to hold at $50 \mathrm{mK}$ for a 24-hour observation period and its cycling is synchronized with that of the $300 \mathrm{mK}$ fridges. The operation of this cooler has been modeled including all conducted loads through structures and wiring, radiative loads, as well as the multiplexer dissipation. Appropriate magnetic shielding is included in our mass budget, as is the copper strapping required to insure that thermal gradients are less than $0.5 \mathrm{mK}$ on the cold stage.

To demonstrate the feasibility of this approach, we have built and are now characterizing a prototype in a cryostat which includes regulated stages at $4.5 \mathrm{~K}$ and $1.7 \mathrm{~K}$. As Figure 9 shows, we are able to regulate the cycling of the ${ }^{3} \mathrm{He}$ coolers and the salt pill to operate long-term with $7 \mathrm{~mW}$ and $3 \mathrm{~mW}$ dissipation at $4.5 \mathrm{~K}$ and $1.7 \mathrm{~K}$, respectively. Further information is available in Prouvé et al. article in Cryogenics, currently in press.

\section{ACKNOWLEDGMENTS}

We thank Jeremy Hodis for help with measuring the WaFIRS spectrometer prototype. This work has been supported in part with NASA ROSES grant \#65277804.04.01.01.01.

\section{REFERENCES}

1. Hubickyj, O., Bodenheimer, P., and Lissauer, J. J., "Accretion of the gaseous envelope of Jupiter around a 510 Earth-mass core," Icarus 179, 415-431 (Dec. 2005). 
2. Takeuchi, T. and Lin, D. N. C., "Attenuation of Millimeter Emission from Circumstellar Disks Induced by the Rapid Dust Accretion," ApJ 623, 482-492 (Apr. 2005).

3. Ward, W. R., "Survival of Planetary Systems," ApJL 482, L211+ (June 1997).

4. Kominami, J. and Ida, S., "The Effect of Dissipating Gas Disk on Terrestrial Planet Formation," in [Bulletin of the American Astronomical Society], Bulletin of the American Astronomical Society 34, 941-+ (Sept. 2002).

5. Oliver, S. J., et al., "The Herschel Multi-tiered Extragalactic Survey: HerMES," MNRAS , 3269 (July 2012).

6. Armus, L., et al., "Observations of Ultraluminous Infrared Galaxies with the Infrared Spectrograph on the Spitzer Space Telescope. II. The IRAS Bright Galaxy Sample," ApJ 656, 148-167 (Feb. 2007).

7. Farrah, D., Bernard-Salas, J., Spoon, H. W. W., Soifer, B. T., Armus, L., Brandl, B., Charmandaris, V., Desai, V., Higdon, S., Devost, D., and Houck, J., "High-Resolution Mid-Infrared Spectroscopy of Ultraluminous Infrared Galaxies," ApJ 667, 149-169 (Sept. 2007).

8. Papovich, C., Rudnick, G., Le Floc'h, E., van Dokkum, P. G., Rieke, G. H., Taylor, E. N., Armus, L., Gawiser, E., Huang, J., Marcillac, D., and Franx, M., "Spitzer Mid- to Far-Infrared Flux Densities of Distant Galaxies," ApJ 668, 45-61 (Oct. 2007).

9. Daddi, E., et al., "Multiwavelength Study of Massive Galaxies at z 2. II. Widespread Compton-thick Active Galactic Nuclei and the Concurrent Growth of Black Holes and Bulges," ApJ 670, 173-189 (Nov. 2007).

10. Soltan, A., "Masses of quasars," MNRAS 200, 115-122 (July 1982).

11. Yu, Q. and Tremaine, S., "Observational constraints on growth of massive black holes," MNRAS 335, 965-976 (Oct. 2002).

12. Hopkins, P. F., Hernquist, L., Cox, T. J., Robertson, B., and Krause, E., "An Observed Fundamental Plane Relation for Supermassive Black Holes," ApJ 669, 67-73 (Nov. 2007).

13. Wang, W.-H., Barger, A. J., and Cowie, L. L., "Ultradeep Near-Infrared Observations of Goods 850-5," ApJ 690, 319-329 (Jan. 2009).

14. Egami, E., Rieke, G. H., Fadda, D., and Hines, D. C., "A Large Mass of $\mathrm{H}_{2}$ in the Brightest Cluster Galaxy in Zwicky 3146," ApJL 652, L21-L24 (Nov. 2006).

15. Ogle, P., Antonucci, R., Appleton, P. N., and Whysong, D., "Shocked Molecular Hydrogen in the $3 \mathrm{C} 326$ Radio Galaxy System," ApJ 668, 699-707 (Oct. 2007).

16. Appleton, P. N., Xu, K. C., Reach, W., Dopita, M. A., Gao, Y., Lu, N., Popescu, C. C., Sulentic, J. W., Tuffs, R. J., and Yun, M. S., "Powerful High-Velocity Dispersion Molecular Hydrogen Associated with an Intergalactic Shock Wave in Stephan's Quintet," ApJL 639, L51-L54 (Mar. 2006).

17. Bradford, C. M., et al., "WaFIRS: A waveguide far-IR spectrometer: Enabling spectroscopy of high-z galaxies in the far-IR and submillimeter," in [IR Space Telescopes and Instruments], Mather, J. C., ed., Proceedings of the SPIE 4850, 1137-1148 (Mar. 2003).

18. Bradford, C. M., Aguirre, J. E., Aikin, R., Bock, J. J., Earle, L., Glenn, J., Inami, H., Maloney, P. R., Matsuhara, H., Naylor, B. J., Nguyen, H. T., and Zmuidzinas, J., "The Warm Molecular Gas around the Cloverleaf Quasar," ApJ 705, 112-122 (Nov. 2009).

19. Beyer, A. D., Kenyon, M. E., Echternach, P. M., Eom, B., Bueno, J., Day, P. K., Bock, J. J., and Bradford, C. M., "Characterizing SixNy absorbers and support beams for far-infrared/submillimeter transition-edge sensors," in [Society of Photo-Optical Instrumentation Engineers (SPIE) Conference Series], Society of Photo-Optical Instrumentation Engineers (SPIE) Conference Series $\mathbf{7 7 4 1}$ (July 2010).

20. Reintsema, C. D., Beyer, J., Nam, S. W., Deiker, S., Hilton, G. C., Irwin, K., Martinis, J., Ullom, J., Vale, L. R., and MacIntosh, M., "Prototype system for superconducting quantum interference device multiplexing of large-format transition-edge sensor arrays," Review of Scientific Instruments 74(10), 4500-4508 (2003).

21. Duband, L., Clerc, L., Ercolani, E., Guillemet, L., and Vallcorba, R., "Herschel flight model sorption coolers," Cryogenics 48, 95-105 (2008).

22. Shirron, P., Dipirro, M., Panek, J., Kelley, R., Mitsuda, K., Fujimoto, R., Hirabayashi, M., and McCammon, D., "The astro-e2/xrs-2 helium insert system," Nuc. Inst. and Meth. in Phys. A 559, 666-668 (2006). 